\title{
Late mortality and causes of death among long-term survivors after autologous hematopoietic stem cell transplantation
}

Minako lida', Hideki Nakasone ${ }^{2}$, Takuya Yamashita ${ }^{3}$, Masami Inoue $^{4}$, Yasushi Ishida ${ }^{5}$, Hitoji Uchiyama ${ }^{6}$, Yuta Katayama ${ }^{7}$, Toshihiro Miyamoto ${ }^{8}$, Satoshi Yoshioka ${ }^{9}$, Souichi Shiratori ${ }^{10}$, Takehiko Mori ${ }^{11}$, Masashi Sawa ${ }^{12}$, Yasuhiro Sugio ${ }^{13}$, Takahiro Fukuda ${ }^{14}$, Tatsuo Ichinohe ${ }^{15}$, Yoshiko Atsuta ${ }^{16}$, Yoshihiro Inamoto ${ }^{14}$, for the Late Effect and Quality of Life Working Group of the Japan Society for Hematopoietic Cell Transplantation

${ }^{1}$ Department of Promotion for Blood and Marrow Transplantation, Aichi Medical University School of Medicine, Nagakute, Japan, ${ }^{2}$ Division of Hematology, Saitama Medical Center, Jichi Medical University, Saitama, Japan, ${ }^{3}$ Department of Hematology, St. Luke' s International Hospital, Tokyo, Japan, ${ }^{4}$ Department of Hematology/Oncology, Osaka Women's and Children's Hospital, Osaka, Japan, ${ }^{5}$ Department of Pediatrics, Ehime Prefectural Central Hospital, Matsuyama, Japan, ${ }^{6}$ Department of Hematology, Japanese Red Cross Kyoto Daiichi Hospital, Kyoto, Japan, ${ }^{7}$ Department of Hematology, Hiroshima Red Cross Hospital \& Atomic-bomb Survivors Hospital, Hiroshima, Japan, ${ }^{8}$ Medicine and Biosystemic Science, Graduate School of Medical Sciences, Kyushu University, Fukuoka, Japan, ${ }^{9}$ Department of Hematology, Kobe City Medical Center General Hospital, Kobe, Japan, ${ }^{10}$ Department of Hematology, Hokkaido University Hospital, Sapporo, Japan, ${ }^{11}$ Division of Hematology, Department of Medicine, Keio University School of Medicine, Tokyo, Japan, ${ }^{12}$ Department of Hematology and Oncology, Anjo Kosei Hospital, Anjo, Japan, ${ }^{13}$ Department of Internal Medicine, Kitakyushu City Hospital Organization, Kitakyushu Municipal Medical Center, Kitakyushu, Japan, ${ }^{14}$ Department of Hematopoietic Stem Cell Transplantation, National Cancer Center Hospital, Tokyo, Japan, ${ }^{15}$ Department of Hematology and Oncology, Research Institute for Radiation Biology and Medicine, Hiroshima University, Hiroshima, Japan, ${ }^{16}$ Japanese Data Center for Hematopoietic Cell Transplantation/Department of Healthcare Administration, Nagoya University Graduate School of Medicine, Nagoya, Japan,

Abstract

By evaluating risks of late mortality and causes of death among long-term survivors after autologous hematopoietic stem cell transplantation (HSCT) in Japan, we clarified what we should focus on during follow-up to reduce them. The study cohort included 6,780 patients who had survived for $\geq 2$ years after the first autologous HSCT performed from 1974 to 2012 for hematological diseases. With a median follow-up of 6.0 years among survivors, overall survival probabilities at 5 and 10 years after HSCT were $92 \%$ and $83 \%$, respectively. Eight hundred thirty deaths occurred: 451, recurrent primary diseases; 87 , subsequent solid cancers; 57 , subsequent hematological malignancies; 55, infections; 41, respiratory diseases; 19, cardiovascular diseases; 15, liver diseases; 10, neurological diseases; and 7, kidney/genitourinary diseases (Except small numbers of other causes and missing). According to the log-rank test, the risk of overall mortality was remarkably increased among HSCT recipients compared with the that in the general Japanese population (observed/expected ratio $[\mathrm{O} / \mathrm{E}]=5.4 ; 95 \%$ confidence interval $[\mathrm{Cl}]$, 5.0-5.8). The risks of cause-specific mortality increased with infection ( $\mathrm{O} / \mathrm{E}=6.8 ; 95 \% \mathrm{Cl}, 5.1-8.8)$, subsequent solid cancers $(\mathrm{O} / \mathrm{E}=1.4 ; 95 \% \mathrm{Cl}, 1.1-1.7)$, subsequent hematological malignancies $(\mathrm{O} / \mathrm{E}=14.3 ; 95 \% \mathrm{Cl}, 10.8-18.5)$, kidney/genitourinary diseases $(\mathrm{O} / \mathrm{E}=3.4 ; 95 \% \mathrm{Cl}, 1.4-7.1)$, respiratory disease $(\mathrm{O} / \mathrm{E}=9.0 ; 95 \% \mathrm{Cl}, 6.5-1.2)$, and liver diseases $(\mathrm{O} / \mathrm{E}=2.6 ; 95 \% \mathrm{Cl}, 1.4-4.2)$. Long-term survivors after autologous $\mathrm{HSCT}$ are at an increased risk of death due to secondary cancers, infections, and any organ diseases as well as recurrence compared to the general population. When monitoring these patients in the outpatient clinic, it is important for physicians to predict a change in the patient' $s$ condition and to start treatment earlier.

Key words: Late mortality, Cause of death, Long-term survivors, Autologous HSCT

Submitted October 18, 2018; Accepted December 14, 2019; Published online February 25, 2020; Issued online February 25, 2020

Correspondence: Minako Iida, Department of Promotion for Blood and Marrow Transplantation, Aichi Medical University School of Medicine, 1-1, Yazakokarimata, Nagakute, Aichi 480-1195, Japan, E-mail: miida@aichi-med-u.ac.jp 


\section{Introduction}

The number of autologous hematopoietic stem cell transplantation (HSCT) is continuously increasing ${ }^{1}$, and the proportion of long-term survivors after HSCT is expected to increase with the advancement of transplantation and other medical techniques. The risk of developing diseases after autologous HSCT is high in survivors compared to that in the general population owing to damage caused to body organs by consumption of numerous drugs for conditioning chemotherapy and prevention and treatment of complications, primary diseases, which necessitated HSCT, and HSCT itself, although patients with malignant diseases do not experience recurrence. In this study, we evaluated the risks of late mortality and causes of death among long-term survivors after autologous HSCT in Japan and identified important guidelines for predicting and reducing the occurrence of late complications in the out-patient clinic.

\section{Patients and Methods}

\section{Patients and data collection}

The patient clinical data were collected by the Japan Society for Hematopoietic Cell Transplantation (JSHCT)/Japanese Data Center for Hematopoietic Cell Transplantation (JDCHCT) using the Transplant Registry Unified Management Program (TRUMP) ${ }^{2,3}$. The study cohort included 6,780 patients characterized by progression-free survival for $\geq 2$ years after the first autologous HSCT, performed from 1974 to 2012 for hematological diseases. This study was approved by the data management committees of the JSHCT and by the institutional review board of the Ethical Committee of Aichi Medical University. JSHCT/JDCHCT observational research protocol requires written informed consents from patients for use of their data for research purposes. Opt-out was permitted, in accordance with the Ethical Guidelines, for patients who received HSCT before May 2017, and those who declined permission for participation in research were not included in the study.

\section{Classification of causes of death}

Causes of death were classified according to the International Classification of Diseases, Tenth Revision ${ }^{4}$. In TRUMP, the causes of death were reported by transplant physicians based on two categories of primary and secondary. If causes of death were listed as " other, " detailed information was written in the free description column. All of these were reviewed and re-classified according the definition of International Classification of Diseases by six members in this Working Group (WG) (Supplemental Table). All cases described as "relapse or progression of underlying disease" under either the main cause or secondary cause categories were classified as "recurrent disease". In addition, members carefully distinguished between infection and respiratory disease in the classification of causes of death in TRUMP. This classification was the same as that used in previously-reported research by the $\mathrm{WG}^{5}$.

\section{Statistical analysis}

The standard mortality ratio (SMR) was calculated by dividing the number of deaths among patients with HSCT (observed number) by the number of expected deaths in the age-, sex-, and year-matched general population (expected number). The mortality rates in the general population were obtained from the database of the Health, Labour and Welfare Statistics Association in Japan. The 95\% confidence intervals (CIs) for the SMR were calculated based on the assumption that the observed numbers of deaths followed a Poisson distribution. Overall survival was estimated from HSCT to death by any cause using the Kaplan-Meier method. The logrank test was used to draw comparisons between the groups. A two-sided P-value $<0.05$ was considered statistically significant. Data were analyzed using STATA, version 10 statistical software (STATA Corp., College Station, TX, USA).

\section{Results}

Patient characteristics are summarized in Table 1. Patients' median age at HSCT was 49 years (range, 0-78 years), and $70 \%$ of them were $\geq 40$ years of age. Ninetyfive percent of the transplant sources were peripheral blood (PB) including a very small number of bone marrow plus PB. As for the patients' diseases, except for the small number of non-malignant diseases $(n=213)$, most were hematological malignancies such as non-Hodgkin lymphoma (NHL) $(\mathrm{n}=4,191)$, multiple myeloma $(\mathrm{MM}) /$ plasma cell disorder $(\mathrm{n}=805)$, acute myeloid leukemia (AML) $(\mathrm{n}=795)$, Hodgkin lymphoma (HL) $(\mathrm{n}=422)$, and acute lymphoblastic leukemia (ALL) $(\mathrm{n}=354)$.

The overall survival probability at 10 years after HSCT among 2-year survivors was $83 \%$ (95\% CI, 81\%-84\%), with a median follow-up of 6.0 years after HSCT (range, 2.0-27.6 years) (Figure 1). Eight hundred thirty patients died 2 years after HSCT. The most frequent cause of death was recurrent disease $(n=451,54 \%)$, followed by new malignancies $(\mathrm{n}=144,17 \%)$ including solid cancers $(\mathrm{n}=87)$ and hematological malignancies $(\mathrm{n}=57)$, infections $(n=55,6.6 \%)$, respiratory diseases $(n=41,4.9 \%)$, cardiovascular diseases $(n=19,2.3 \%)$, liver diseases $(\mathrm{n}=15,1.8 \%)$, and neurological diseases $(\mathrm{n}=10,1.2 \%)$ (Figure 2). The difference in the causes of death in two groups based on the transplant year, before 2005 and 
Table 1. Characteristics of the disease-free survivors who survived more than two years after autologous transplantation

\begin{tabular}{|c|c|c|c|}
\hline & & \multicolumn{2}{|c|}{$\mathrm{n}=6,780$} \\
\hline & & $\mathrm{n}$ & $(\%)$ \\
\hline \multirow[t]{5}{*}{ Age (years) } & $0-15$ & 564 & 8.3 \\
\hline & $16-30$ & 852 & 12.6 \\
\hline & $31-45$ & 1,326 & 19.5 \\
\hline & $46-60$ & 2,858 & 42.2 \\
\hline & $61-$ & 1,180 & 17.4 \\
\hline \multicolumn{2}{|c|}{ Median age (range) (years) } & \multicolumn{2}{|c|}{$49(0-78)$} \\
\hline \multirow[t]{2}{*}{ Sex } & Male & 3,897 & 57.5 \\
\hline & Female & 2,883 & 42.5 \\
\hline \multirow[t]{5}{*}{ Transplant year } & -1990 & 98 & 1.5 \\
\hline & 1991-1995 & 699 & 10.3 \\
\hline & 1996-2000 & 1,484 & 21.9 \\
\hline & $2001-2005$ & 1,770 & 26.1 \\
\hline & 2006-2012 & 2,729 & 40.2 \\
\hline \multirow[t]{2}{*}{ Source } & Bone marrow $(\mathrm{BM})$ & 337 & 5.0 \\
\hline & Peripheral blood (PB) (including BM+PB) & 6,443 & 95.0 \\
\hline \multirow[t]{6}{*}{ Diseases } & Non-Hodgkin lymphoma & 4,191 & 61.8 \\
\hline & Multiple myeloma/Plasma cell disorder & 805 & 11.9 \\
\hline & Acute myeloid leukemia & 795 & 11.7 \\
\hline & Hodgkin lymphoma & 422 & 6.2 \\
\hline & Acute lymphoblastic leukemia & 354 & 5.2 \\
\hline & Others* & 213 & 3.2 \\
\hline \multirow[t]{2}{*}{ Total body irradiation } & No & 5,818 & 85.8 \\
\hline & Yes & 468 & 6.9 \\
\hline \multirow[t]{2}{*}{ Etoposide } & No & 1,790 & 26.4 \\
\hline & Yes & 4,631 & 68.3 \\
\hline \multirow[t]{2}{*}{ Cyclophosphamide } & No & 2,750 & 40.6 \\
\hline & Yes & 3,665 & 54.1 \\
\hline \multirow[t]{2}{*}{ Ranimustine } & No & 3,504 & 55.0 \\
\hline & Yes & 2,866 & 45.0 \\
\hline \multirow[t]{2}{*}{ Melphalan } & No & 3,743 & 55.2 \\
\hline & Yes & 2,663 & 39.3 \\
\hline \multirow[t]{2}{*}{ Carboplatin } & No & 4,277 & 67.1 \\
\hline & Yes & 2,093 & 32.9 \\
\hline \multirow[t]{2}{*}{ Survival after 2 years } & Alive & 5,950 & 87.8 \\
\hline & Death & 830 & 12.2 \\
\hline \multicolumn{2}{|c|}{ Observation period on survival（years） } & 2.0-27.6 & (6.0) \\
\hline
\end{tabular}

*Other includes bleeding, sudden death and multiorgan failure.

after 2006, was analyzed. There was no significant difference between the two groups, with new malignancies as the predominant cause of death in both groups, followed by infections and respiratory diseases (data not shown). The SMR for any cause of death in survivors after HSCT was 5.4 (95\% CI, 5.0-5.8). The SMRs for each cause of death were elevated with subsequent hematological malignancies (SMR 14.3; 95\% CI, 10.8-18.5), respiratory diseases (SMR, 9.0; 95\% CI, 6.5-12.2), infections (SMR, 6.8; 95\% CI, 5.1-8.8), kidney/genitourinary diseases (SMR, 3.4; 95\% CI, 1.4-7.1), liver diseases (SMR, 2.6; 95\% CI, 1.4-4.2), and non-hematological new malignancies（SMR, 1.4; 95\% CI, 1.1-1.7） (Table 2).

\section{Discussion}

According to the present study's findings, long-term survivors after autologous HSCT in Japan have various late complications and a higher risk of death due to any cause compared to the general population. This result shows that it is important to predict various late complications and to provide early prevention and treatment to patients for many years after HSCT.

New malignancies, especially hematological malignancies, were the most frequent cause of mortality, excluding recurrence, after autologous HSCT. Reported by Pedersen-Bjergaard et al and Miller et $\mathrm{al}^{6,7}$, high-risk incidents of therapy-related AML/MDS（t-AML/MDS） 


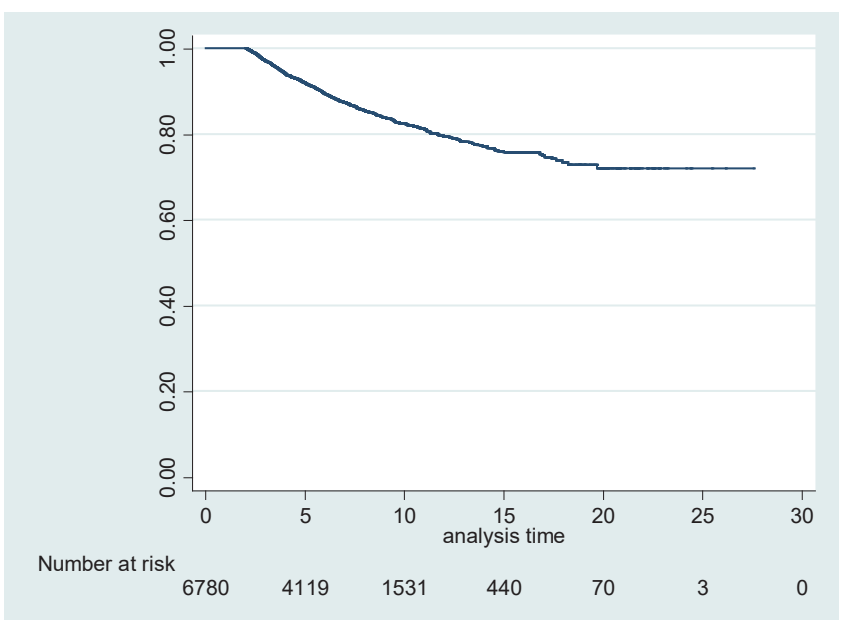

Figure 1. Probabilities of overall survival among recurrence-free survivors over time

The probability of overall survival among recurrence-free survivors at 2 years after autologous hematopoietic stem cell transplantation was $82.6 \%$ at 12 years.

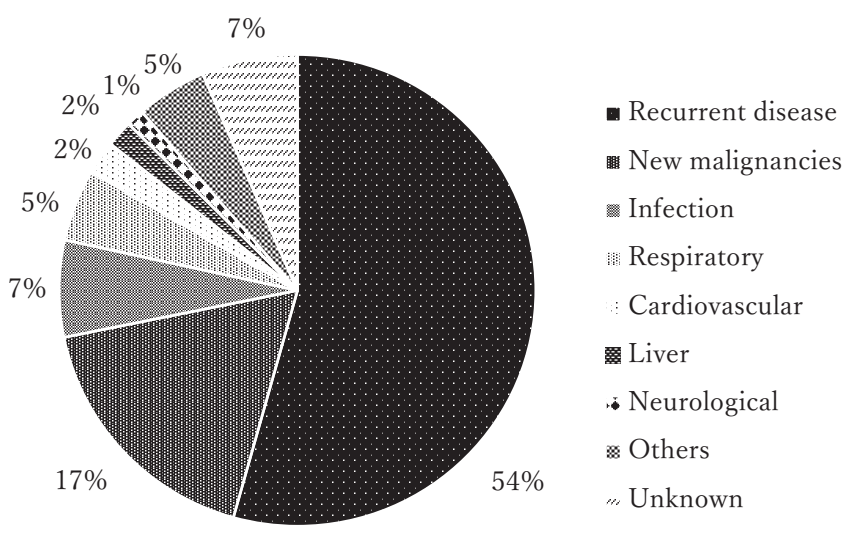

Figure 2. Frequency of causes of death

Other causes of death include kidney and genitourinary diseases, external (accidental) injuries, endocrine/diabetes, digestive tract disorders, suicide, bleeding, sudden death, and multiorgan failure.

Table 2. Standard mortality ratios (observed/expected ratio) for each cause of death among the patients who survived more than two years after transplantation

\begin{tabular}{|c|c|c|c|c|}
\hline \multirow{3}{*}{$\begin{array}{l}\text { Number of patients } \\
\text { Person-years at risk } \\
\text { Cause of Death }\end{array}$} & \multicolumn{4}{|c|}{6,780} \\
\hline & \multicolumn{4}{|c|}{$34,582.75$} \\
\hline & 0 & $E$ & SMR & $95 \% \mathrm{Cl}$ \\
\hline Any cause & 830 & 153.9 & $5.4^{*}$ & $(5.0-5.8)$ \\
\hline New malignancy & 144 & 66.2 & $2.2^{*}$ & $(1.8-2.6)$ \\
\hline Non-hematological malignancies & 87 & 62.3 & $1.4^{*}$ & $(1.1-1.7)$ \\
\hline Hematological malignancies & 57 & 4.0 & $14.3^{*}$ & $(10.8-18.5)$ \\
\hline Infection & 55 & 8.1 & $6.8^{*}$ & $(5.1-8.8)$ \\
\hline Respiratory & 41 & 4.6 & $9.0^{*}$ & $(6.5-12.2)$ \\
\hline Cardiovascular & 19 & 21.9 & 0.9 & $(0.5-1.4)$ \\
\hline Liver & 15 & 5.8 & $2.6^{*}$ & $(1.4-4.2)$ \\
\hline Neurological & 10 & 16.1 & 0.6 & $(0.3-1.1)$ \\
\hline Kidney/Genitourinary & 7 & 2.0 & $3.4^{*}$ & $(1.4-7.1)$ \\
\hline External (Accidental) & 7 & 8.2 & 0.9 & $(0.3-1.8)$ \\
\hline Endocrine/Diabetes & 3 & 3.0 & 1.0 & $(0.2-2.9)$ \\
\hline Digestive & 1 & 2.7 & 0.4 & $(0.0-2.0)$ \\
\hline Suicide & 1 & 9.7 & 0.1 & $(0.0-0.6)$ \\
\hline Mental & 0 & 0.3 & 0.0 & \\
\hline Skin & 0 & 0.1 & 0.0 & \\
\hline Muscle & 0 & 0.6 & 0.0 & \\
\hline Pregnancy & 0 & 0.0 & & \\
\hline Homicide & 0 & 0.1 & 0.0 & \\
\hline Recurrent disease & 451 & - & & \\
\hline Other & 21 & - & & \\
\hline Sudden death & 4 & - & & \\
\hline Multiorgan failure & 1 & - & & \\
\hline Unknown/data missing & 54 & - & & \\
\hline
\end{tabular}

$\mathrm{O}$, number of observed death; E, number of expected death; SMR, standard mortality ratio; $\mathrm{Cl}$, confidence interval; *, significantly higher risk compared to general population where lower limit of $95 \% \mathrm{Cl}$ exceeds 1.0 . 
are well known. They proposed that accumulative alkylating agents used in the preconditioning regimen are one of the biggest risk factors for this clinical condition. We found that many patients were exposed to alkylating agents, such as cyclophosphamide, ranimustine (MCNU), melphalan, and carboplatin, as preconditioning agents in this cohort, and this finding supports that of the previous reports. Use of alternative agents for the conditioning regimen might reduce the risk of t-AML/MDS. However, purine analogs, such as fludarabine, stand out as the risk factor of secondary hematological malignancy after $\mathrm{HSCT}^{8,9}$. Although we had a small number of patients who received purine analogs in this cohort, it is necessary for us to consider not only the alkylating agents but also the risk of secondary hematological malignancies in long-term survivors who received purine analogues.

Older age is one of the risk factors that affects the development of t-AML/MDS after autologous transplantation ${ }^{8,10,11}$. The fact that more than $60 \%$ of the patients in this cohort were 46 years or older supports this finding. As the number of patients with autologous HSCT in Japan has increased in recent years, especially in elderly patients older than 60 years of age ${ }^{12}$, more cautious posttransplant follow-up including close monitoring of complete blood cell count changes will be needed for early detection of t-AML/MDS not only in this age group but also for all patients with other identified risk factors ${ }^{8,10}$.

In this analysis, respiratory disease was the third leading cause of death after recurrence and secondary malignancy. Many patients in this category died of idiopathic interstitial pneumonia or respiratory failure (data not shown). Similar to the case with t-AML/MDS, antineoplastic agents often cause pulmonary disorders. Cyclophosphamide, which is commonly administered in autologous HSCT is one of the most common precipitating drugs for interstitial lung diseases ${ }^{13}$. Although there are many reports on pulmonary toxicity caused by carmustine (BCNU) used for high-dose chemotherapy and autologous $\mathrm{HSCT}^{13-15}$ an MCNU-containing regimen is often used for preconditioning of autologous HSCT in Japan, and its pulmonary toxicity is well known ${ }^{16,17}$. On the other hand, every drug other than chemotherapeutic agents, such as antibiotics or nonsteroidal anti-inflammatory drugs, which are commonly used to treat complications after HSCT, might cause pulmonary diseases ${ }^{18-20}$. Symptoms of respiratory diseases, such as cough, sputum, and shortness of breath, are relatively easy for patients to understand, and immediate examination of arterial blood gas analysis and chest radiography as well as chest computed tomography for patients reporting such symptoms is important. Early detection of respiratory diseases and their treatment can be expected to reduce the risk of death.

Among infections after autologous HSCT, except for bacterial and fungal infections, which are carefully monitored, the risk of viral infection associated with immune reconstitution after HSCT is widely recognized ${ }^{21}$. Reusser et al suggested the significance of a consecutive measurement of the titer of cytomegalovirus antigen ${ }^{22}$, and Schuchter et al pointed out that patients with HL and NHL are at a high risk of varicella-zoster virus infection after autologous $\mathrm{HSCT}^{23}$. The viral antigen titer can detect abnormalities before the development of symptoms, making it possible to initiate earlier administration of antiviral agents, such as acyclovir or foscarnet, and reduce mortality from viral infection.

Recurrence of HL, NHL, and MM is the most commonly-encountered conditions among long-term survivors after autologous HSCT, and various methods are suggested to predict recurrence. Hill et $a l^{24}$ reported that the risk for recurrence in patients with diffuse large B-cell lymphoma with an advanced stage at HSCT was higher than that in complete remission. Sirohi et al proposed the use of the Hasenclever index, which was designed as a prognostic score for patients with $\mathrm{HL}^{25}$ to predict the risk for recurrence after autologous $\mathrm{HSCT}^{26}$. Smeltzer et al suggested that by using fluorodeoxyglucose-positron emission tomography (PET) as a relapse risk assessment tool of HL, earlier salvage chemotherapy for PET-positive patients after HSCT will improve the outcome ${ }^{27}$.

Along with prediction of the risk for recurrence, new prophylactic treatment against recurrence after autologous HSCT has been recommended. Moskowitz et al reported that they could reduce the risk for recurrence by administrating brentuximab vedotin as a consolidation therapy to patients with refractory HL after autologous $\mathrm{HSCT}^{28}$. In addition to planned tandem HSCT, which has shown a high degree of availability for patients with MM since the 2000s, we found many reports regarding the benefits of administrating new agents, such as thalidomide and lenalidomide, as maintenance therapy after HSCT in MM to prolong survival ${ }^{29,30}$.

In conclusion, patients after autologous HSCT can expect long-term survival, but the number of patients who develop late complications will increase. When patients are followed up with after autologous HSCT in outpatient clinics, it is necessary for physicians to predict late complications and to perform appropriate testing and therapeutic interventions without delay. If a long-term survivor lives far from the transplant facility, immediate consultation might be difficult; educating the transplant patients regarding the risk of late-onset complications and the importance of immediate consultation should also be emphasized by the physicians. 


\section{Acknowledgments}

This work was supported in part by the Practical Research Project for Allergic Diseases and Immunology (Research Technology of Medical Transplantation) from Japan Agency for Medical Research and Development, AMED under Grant Number 19ek0510023h0002.

\section{Author's Contributions}

MI, HN, TY, MI, YI, YI, and YA contributed to the design of the study. HU, YK, TM, SY, SS, TM, MS, YS, and TF participated in data collection. TI and MI were responsible for data management. MI and YA analyzed the results and wrote the paper.

\section{Conflict of Interest}

Department of Promotion for Blood and Marrow Transplantation, Aichi Medical University, where Iida belongs, has received donations from Kyowa Kirin Co., Ltd., JCR Pharmaceuticals Co., Ltd., Clinigen K. K., Astellas Pharma Inc., Genzyme Japan K. K., CHUGAI PHARMACEUTICAL CO., LTD., Janssen Pharmaceutical K. K., and Sanofi K. K.. Other authors have no conflict of interest to declare. Disclosure forms provided by the authors are available here.

\section{References}

1. Gratwohl A, Pasquini MC, Aljurf M, Atsuta Y, Baldomero H, Foeken L, et al. One million haemopoietic stem-cell transplants: a retrospective observational study. The Lancet Haematology. 2015; 2: e91-e100.

2. Atsuta Y, Suzuki R, Yoshimi A, Gondo H, Tanaka J, Hiraoka A, et al. Unification of hematopoietic stem cell transplantation registries in Japan and establishment of the TRUMP System. Int J Hematol. 2007; 86: 269-74.

3. Atsuta Y. Introduction of Transplant Registry Unified Management Program 2(TRUMP2): scripts for TRUMP data analyses, part I (variables other than HLA-related data). Int J Hematol. 2016; 103: 3-10.

4. WHO. World Health Organization. List of Official ICD-10 Updates. 2014 WHO, 2014, World Health Organization. List of Official ICD-10 Updatesr. https: //www.who.int/classifica tions/icd/icd10updates/en/[Accessed September 28, 2019]

5. Atsuta Y, Hirakawa A, Nakasone H, Kurosawa S, Oshima K, Sakai R, et al. Late Mortality and Causes of Death among Long-Term Survivors after Allogeneic Stem Cell Transplantation. Biol Blood Marrow Transplant. 2016; 22: 1702-9.

6. Pedersen-Bjergaard J, Andersen MK, Christiansen DH. Therapy-related acute myeloid leukemia and myelodysplasia after high-dose chemotherapy and autologous stem cell transplanta- tion. Blood. 2000; 95: 3273-9.

7. Miller JS, Arthur DC, Litz CE, Neglia JP, Miller WJ, Weisdorf DJ. Myelodysplastic syndrome after autologous bone marrow transplantation: an additional late complication of curative cancer therapy. Blood. 1994; 83: 3780-6.

8. Micallef IN, Lillington DM, Apostolidis J, Amess JA, Neat M, Matthews J, et al. Therapy-related myelodysplasia and secondary acute myelogenous leukemia after high-dose therapy with autologous hematopoietic progenitor-cell support for lymphoid malignancies. J Clin Oncol. 2000; 18: 947-55.

9. Orchard JA, Bolam S, Oscier DG. Association of myelodysplastic changes with purine analogues. Br J Haematol. 1998; 100: 677-9.

10. Ge I, Saliba RM, Maadani F, Popat UR, Qazilbash MH, Ravi Pingali S, et al. Patient age and number of apheresis days may predict development of secondary myelodysplastic syndrome and acute myelogenous leukemia after high-dose chemotherapy and autologous stem cell transplantation for lymphoma. Transfusion. 2017; 57: 1052-7.

11. Kalaycio M, Rybicki L, Pohlman B, Sobecks R, Andresen S, Kuczkowski E, et al. Risk factors before autologous stem-cell transplantation for lymphoma predict for secondary myelodysplasia and acute myelogenous leukemia. J Clin Oncol. 2006; 24: $3604-10$.

12. JDCHCT, The Japanese Data Center for Hematopoietic Cell Transplantation; Activities and Outcomes of Hematopoietic Cell Transplantation in Japan 2018 Summary Slide, 2018. http: //www.jdchct.or.jp/en/data/slide/2018/[Accessed September 28, 2019]

13. Wilczynski SW, Erasmus JJ, Petros WP, Vredenburgh JJ, Folz RJ. Delayed pulmonary toxicity syndrome following high-dose chemotherapy and bone marrow transplantation for breast cancer. Am J Respir Crit Care Med. 1998; 157: 565-73.

14. Todd NW, Peters WP, Ost AH, Roggli VL, Piantadosi CA. Pulmonary drug toxicity in patients with primary breast cancer treated with high-dose combination chemotherapy and autologous bone marrow transplantation. Am Rev Respir Dis. 1993; 147: 1264-70.

15. Bhalla KS, Wilczynski SW, Abushamaa AM, Petros WP, McDonald CS, Loftis JS, et al. Pulmonary toxicity of induction chemotherapy prior to standard or high-dose chemotherapy with autologous hematopoietic support. Am J Respir Crit Care Med. 2000; 161: 17-25.

16. Kato J, Mori T, Yokoyama K, Tsukada Y, Ueda T, Shimizu T, et al. Safety and efficacy of high-dose ranimustine, cytarabine, etoposide and CY (MCVAC) regimen followed by autologous peripheral blood stem cell transplantation for high-risk diffuse large B-cell lymphoma. Bone Marrow Transplant. 2011; 46: 923-8.

17. Kameoka Y, Akagi T, Murai K, Noji H, Kato Y, Sasaki O, et al. Safety and efficacy of high-dose ranimustine (MCNU) containing regimen followed by autologous stem cell transplantation for diffuse large B-cell lymphoma. Int J Hematol. 2018; 108: $510-5$. 
18. Cooper JA, Jr., White DA, Matthay RA. Drug-induced pulmonary disease. Part 2: Noncytotoxic drugs. Am Rev Respir Dis. 1986; 133: 488-505.

19. Cooper JA, Jr., Matthay RA. Drug-induced pulmonary disease. Dis Mon. 1987; 33: 61-120.

20. Camus $P$, Kudoh S, Ebina M. Interstitial lung disease associated with drug therapy. Br J Cancer. 2004; 91 Suppl 2: S18-23.

21. Guillaume T, Rubinstein DB, Symann M. Immune reconstitution and immunotherapy after autologous hematopoietic stem cell transplantation. Blood. 1998; 92: 1471-90.

22. Reusser P, Fisher LD, Buckner CD, Thomas ED, Meyers JD. Cytomegalovirus infection after autologous bone marrow transplantation: occurrence of cytomegalovirus disease and effect on engraftment. Blood. 1990; 75: 1888-94.

23. Schuchter LM, Wingard JR, Piantadosi S, Burns WH, Santos GW, Saral R. Herpes zoster infection after autologous bone marrow transplantation. Blood. 1989; 74: 1424-7.

24. Hill BT, Rybicki L, Bolwell BJ, Smith S, Dean R, Kalaycio M, et al. The non-relapse mortality rate for patients with diffuse large B-cell lymphoma is greater than relapse mortality 8 years after autologous stem cell transplantation and is significantly higher than mortality rates of population controls. Br J Haematol. 2011 ; 152: 561-9.

25. Hasenclever D, Diehl V. A prognostic score for advanced Hodgkin's disease. International Prognostic Factors Project on Advanced Hodgkin's Disease. N Engl J Med. 1998; 339: 150614.

26. Sirohi B, Cunningham D, Powles R, Murphy F, Arkenau T,
Norman A, et al. Long-term outcome of autologous stem-cell transplantation in relapsed or refractory Hodgkin's lymphoma. Ann Oncol. 2008; 19: 1312-9.

27. Smeltzer JP, Cashen AF, Zhang Q, Homb A, Dehdashti F, Abboud CN, et al. Prognostic significance of FDG-PET in relapsed or refractory classical Hodgkin lymphoma treated with standard salvage chemotherapy and autologous stem cell transplantation. Biol Blood Marrow Transplant. 2011; 17: 1646-52.

28. Moskowitz CH, Nademanee A, Masszi T, Agura E, Holowiecki $\mathrm{J}$, Abidi $\mathrm{MH}$, et al. Brentuximab vedotin as consolidation therapy after autologous stem-cell transplantation in patients with Hodgkin's lymphoma at risk of relapse or progression (AETHERA): a randomised, double-blind, placebo-controlled, phase 3 trial. Lancet. 2015; 385: 1853-62.

29. Spencer A, Prince HM, Roberts AW, Prosser IW, Bradstock KF, Coyle L, et al. Consolidation therapy with low-dose thalidomide and prednisolone prolongs the survival of multiple myeloma patients undergoing a single autologous stem-cell transplantation procedure. J Clin Oncol. 2009; 27: 1788-93.

30. Attal M, Lauwers-Cances V, Marit G, Caillot D, Moreau P, Facon T, et al. Lenalidomide maintenance after stem-cell transplantation for multiple myeloma. N Engl J Med. 2012; 366: 1782-91.

https: //doi.org/10.31547/bct-2019-011

Copyright (C) 2020 APBMT. All Rights Reserved. 